\title{
Hospitalization increases while economic status deteriorates in late stages of chronic obstructive pulmonary disease: the Korean National Health and Nutrition Examination Survey for 2007-2015
}

\author{
Hyung Koo Kang ${ }^{1 \#}$, Jae-Woo Jung ${ }^{2 \#}$, Min-Jong Kang ${ }^{3}$, Deog Kyeom Kim ${ }^{4}$, Hayoung Choi ${ }^{5}$, \\ Young Jae Cho ${ }^{6}$, Seung Hun Jang ${ }^{7}$, Chang Hoon Lee ${ }^{8}$, Yeon Mok Oh' ${ }^{9}$ Jisook Park ${ }^{10}$, Jae Yeol Kim ${ }^{2}$
}

${ }^{1}$ Division of Pulmonary and Critical Care Medicine, Department of Internal Medicine, Ilsan Paik Hospital, Inje University College of Medicine, Goyang, Republic of Korea; ${ }^{2}$ Department of Internal Medicine, Chung-Ang University College of Medicine, Seoul, Republic of Korea; ${ }^{3}$ Section of Pulmonary, Critical Care and Sleep Medicine, Department of Internal Medicine, Yale University School of Medicine, New Haven, CT, USA; ${ }^{4}$ Department of Internal Medicine, Seoul National University College of Medicine, SMG-SNU Borame Medical Center, Seoul, Republic of Korea; ${ }^{5}$ Department of Internal Medicine, Hallym University Kangnam Sacred Heart Hospital, Seoul, Republic of Korea; ${ }^{6}$ Division of Pulmonary and Critical Care Medicine, Department of Internal Medicine, Seoul National University College of Medicine, Seoul National University Bungdang Hospital, Seongnam, Republic of Korea; ${ }^{7}$ Division of Pulmonary, Allergy and Critical Care Medicine, Hallym University Sacred Heart Hospital, Anyang, Republic of Korea; ${ }^{8}$ Department of Internal Medicine, Seoul National University College of Medicine, Seoul, Republic of Korea; ${ }^{9}$ Department of Pulmonary and Critical Care Medicine, Asan Medical Center, University of Ulsan College of Medicine, Seoul, Republic of Korea; ${ }^{10}$ Department of Software Convergence, Seoul Women's University College of Interdisciplinary Studies for Emerging Industries, Seoul, Republic of Korea

Contributions: (I) Conception and design: MJ Kang, JY Kim; (II) Administrative support: HK Kang, JY Kim; (III) Provision of study materials or patients: JY Kim; (IV) Collection and assembly of data: J Park; (V) Data analysis and interpretation: JW Jung; (VI) Manuscript writing: All authors; (VII) Final approval of manuscript: All authors.

\#These authors contributed equally to this work and are co-first authors.

Correspondence to: Jae Yeol Kim, MD. Department of Internal Medicine, Chung-Ang University College of Medicine, 102 Heukseok-ro, Dongjak-gu, Seoul, Republic of Korea. Email: jykimmd@cau.ac.kr.

Background: Chronic obstructive pulmonary disease (COPD) is associated with frequent hospitalizations, higher mortality, and healthcare costs. Low-income COPD patients have higher rates of emergency department visits and hospitalization due to COPD exacerbation. However, other causes of admissions and their economic burden have not been well-elucidated.

Methods: We analyzed the Korean National Health and Nutrition Examination Survey (KNHANES) dataset for 2007-2015. The diagnosis and staging of COPD were based on the Global Initiative for Chronic Obstructive Lung Disease (GOLD) guidelines.

Results: Among the 97,622 participants in KNHANES for 2007-2015, we selected 33,963 participants (4,430 with and 29,533 without COPD) aged $\geq 40$ years, who underwent spirometry, and provided the admission history for the previous year. Participants with COPD had a higher admission rate than those without COPD (12.8\% vs. 10.4\%, $\mathrm{P}<0.001)$. The admission rate increased as the stage of COPD advanced from GOLD 1 to GOLD 4 for total causes $(11.5 \%, 13.6 \%, 15.1 \%$, and $25.0 \%$, respectively, $\mathrm{P}<0.001)$ and respiratory illnesses $(0.5 \%, 1.3 \%, 4.6 \%$, and $12.5 \%$, respectively, $\mathrm{P}<0.001)$. The proportion of the lowest quartile household income increased in the late stages of COPD (GOLD 1-4; 35.2\%, 32.1\%, 44.9\%, and $70.8 \%$, respectively, $\mathrm{P}<0.01$ ).

Conclusions: The hospitalization rate increased in advanced COPD, while GOLD stages 3 and 4 were associated with deterioration in economic status.

Keywords: Chronic obstructive pulmonary disease (COPD); hospitalization; severity; economic status

Submitted Oct 13, 2020. Accepted for publication Feb 19, 2021.

doi: $10.21037 /$ jtd-20-2683

View this article at: http://dx.doi.org/10.21037/jtd-20-2683

(C) Journal of Thoracic Disease. All rights reserved. 


\section{Introduction}

Chronic obstructive pulmonary disease (COPD) is a common respiratory disease affecting $\geq 5 \%$ of the population (1). The prevalence of COPD among the population aged $\geq 40$ years ranged between 13.1-14.6\% during 2010-2015 in Korea (2). COPD is currently the fourth leading cause of death in the United States (3). It is the $8^{\text {th }}$ leading cause of death in Korea with a 5 -year mortality rate of $25.4 \%$ (29.9\% in males and $19.1 \%$ in females) (4). Patients with COPD struggle with the disease and the associated financial costs. COPD is a costly disease and the burden increases with increasing prevalence. In 2010, COPD-attributable costs for both direct medical care and absenteeism in the US were estimated USD 36 billion, with an expected rise to USD 49 billion by 2020 (5). The estimated total costs for COPD were approximately 1.245 billion USD in Korea in 2015 and direct non-medical costs, such as nursing costs made up the largest percentage (39\%) (6). Meanwhile, the average number of days of out-patient care and hospitalization for COPD were $40 \pm 36$ and $11 \pm 33$ days, respectively (7). Lost days for care in COPD lead to an inability to work and unemployment. Predictably, an inverse relationship exists between the prevalence of COPD and income levels. COPD prevalence is $1.5-3$ times higher among people with low socioeconomic status than among those with higher status and low-socioeconomic status patients occupy two-thirds of the COPD population despite comprising $<20 \%$ of the general population (8). COPD patients of a lower socioeconomic status tend to have higher rates of emergency room visits and hospitalizations due to acute exacerbations (9). By the way, COPD is linked to several conditions, such as lung cancer, cardiovascular disease, osteoporosis, psychiatric illness, and cognitive dysfunction that may require hospital admission (10). However, the burden of hospitalization for comorbidities has not been well-elucidated in COPD. In addition, the association between the severity of obstruction and the burden of hospitalization rates and costs has not been well-evaluated in COPD.

The present study evaluated the association between economic status and hospitalization rate with the severity of COPD using data from the Korean National Health and Nutrition Examination Survey (KNHANES) collected for 2007-2015. We present the following article in accordance with the STROBE reporting checklist (available at http:// dx.doi.org/10.21037/jtd-20-2683).

\section{Methods}

\section{Korean National Health and Nutrition Examination Survey (KNHANES)}

We analyzed the KNHANES data for 2007-2015. KNHANES is a national surveillance system that has been assessing the health and nutritional status of Koreans annually since 1998. It is an ongoing, population-based, nationwide, cross-sectional survey conducted by the Korea Centers for Disease Control and Prevention (KCDC). KNHANES encompasses approximately 3,800 households across 576 survey areas selected to represent the civilian non-institutionalized South Korean population. Stratified, multistage, and clustered sampling based on National Census Data is used to ensure that survey results represent the general Korean population (11). Results from each year can be merged into a combined database that allows the review of health trends over long periods. All KNHANES survey protocols were approved by the KCDC institutional review board (2015-01-02-6C). Written informed consent was obtained from all the participants and all procedures were conducted in accordance with the Declaration of Helsinki (as revised in 2013).

\section{Diagnosis of COPD}

KNHANES has three component surveys: a health interview, health examination, and nutritional survey. The health examination includes a pulmonary function test (PFT) that is performed in subjects $\geq 40$ years by welltrained technicians following the American Thoracic Society European Respiratory Society criteria for standardization using a dry rolling-seal spirometer (Vmax-2130, SensorMedics, Yorba Linda, CA, USA). The diagnosis of COPD should be based on the post-bronchodilator PFT findings under the criteria of the Global Initiative for Chronic Obstructive Lung Disease (GOLD) guidelines. However, the KNHANES is not specifically designed to the diagnosis of COPD and post-bronchodilator PFT was not available, therefore, pre-bronchodilator PFT findings were used in the present study. When the ratio of forced expiratory volume in one second $\left(\mathrm{FEV}_{1}\right)$ to functional vital capacity (FVC) was $<0.7$, subjects were considered to have COPD. The severity of airflow limitation was classified according to the GOLD criteria (stage I: mild $\mathrm{FEV}_{1} \geq 80 \%$, stage II: moderate $50 \% \leq \mathrm{FEV}_{1}<80 \%$, stage III: severe $30 \% \leq \mathrm{FEV}_{1}$ 
$<50 \%$, and stage IV: very severe $\left.\mathrm{FEV}_{1}<30 \%\right)$.

\section{Information on hospitalization}

The health interview in KNHANES contains a questionnaire used to collect data regarding hospitalization in the previous year such as the number, duration, cost, satisfaction, and reasons for admission. The reasons for hospital admission described by the participants were transcribed using the Korean Informative Classification of Diseases (KOICD) code by attending statisticians. Among others, the KOICD codes for hospitalization for respiratory diseases include acute upper respiratory tract infection (J00-J01, J05-J06), acute pharyngitis/tonsilitis (J02-J03), acute laryngitis/ bronchitis (J04), influenza (J10-J11), pneumonia (J12-J18), acute bronchitis/bronchiolitis (J20-J21), other nasal diseases (J30-J31), chronic sinusitis (J32), other paranasal diseases (J33-J34), chronic tonsilitis/adenoiditis (J35), other upper respiratory diseases (J36-J39), bronchitis/emphysema/other COPDs (J40-J44), asthma (J45-J46), bronchiectasis (J47), pneumoconiosis (J60-J65), and other respiratory conditions (J22 and J66-J99). Moreover, we evaluated hospitalization for gastrointestinal diseases, cardiovascular illnesses, and malignancy according to matched KOICD codes.

\section{Statistical analysis}

Continuous variables were presented as means \pm standard deviations and categorical data as frequencies and percentiles. Intergroup comparisons of continuous variables and categorical data between the two groups were performed with $t$-tests and chi-square tests, respectively. Multiple group analysis among the four stages of COPD GOLD and non-COPD was done with ANOVA. Binary logistic regression analysis for hospitalization with each stage of GOLD was done and adjusted for age, sex, smoking status, and household income. The results were analyzed using SPSS version 16.0 (SPSS Inc., Chicago, IL, USA). Two-tailed analyses were conducted and $\mathrm{P}$ values $<0.05$ were considered significant.

\section{Results}

\section{Study population}

Of the 97,622 participants in KNHANES for 2007-2015, we selected 33,963 participants who were $\geq 40$ years, underwent spirometry and answered the questionnaire for the information on the history of hospital admission in the previous year. Among the 33,963 participants, COPD was diagnosed for 4,430 participants (13.0\%). GOLD 1 (1,997; $45.1 \%)$ and GOLD $2(2,184 ; 49.3 \%)$ constituted most of the COPD population, while GOLD $3(219 ; 4.7 \%)$ and GOLD 4 (24; $0.5 \%)$ constituted a minor portion (Figure 1).

\section{Comparison of characteristics between participants with and without COPD}

Participants with COPD were older and more likely to be male, and ex- or current-smokers than those without COPD. The lowest quartile of household income occurred with a higher prevalence in the COPD group (34.4\% vs. $18.1 \%, \mathrm{P}<0.001)$. Participants with COPD had more major comorbidities than those without COPD (Table 1).

\section{Demographics, pulmonary function, economic status, and comorbidities according to GOLD stage}

As the stage of COPD advanced from GOLD 1 to 4, the current smoking rate increased, while the percent predicted $\mathrm{FEV}_{1}$ decreased as expected. The proportion of the lowest quartile of household income increased significantly in the late stages of COPD (GOLD 1-4; 35.2\%, 32.1\%, 44.9\%, and $70.8 \%$, respectively, $\mathrm{P}<0.01$ ) (Table 2 ).

\section{The rate, number, and causes of hospitalization}

Among the whole study population, 3,647 participants $(10.7 \%)$ had been hospitalized in the previous year. Most $(3,131 / 3,647,85.9 \%)$ had been admitted once, while others had been hospitalized multiple times (twice in $376 / 3,647$, $10.3 \%$; three times in $87 / 3,647,2.4 \%$; more than three times in $52 / 3,647,1.4 \%$ ). Of the 4,430 participants with COPD, 568 had been hospitalized, while 3,070 of the 29,533 participants without COPD had a history of hospitalization in the previous year $(12.8 \%$ vs. $10.4 \%, \mathrm{P}<0.01)$. For causes of admission, patients with COPD had a higher admission rate than those without COPD for respiratory illnesses (1.1\% vs. $0.4 \%, \mathrm{P}<0.01)$, gastrointestinal diseases $(1.3 \%$ vs. $0.8 \%, \mathrm{P}<0.5)$, and cardiovascular causes $(1.3 \%$ vs. $0.8 \%, \mathrm{P}<0.05)$. Patients with COPD had been hospitalized more frequently than those without COPD for all causes $(0.165 \pm 0.532$ vs. $0.125 \pm 0.432, \mathrm{P}<0.01)$, respiratory causes $(0.018 \pm 0.246$ vs. $0.005 \pm 0.076, \mathrm{P}<0.05)$, gastrointestinal causes $(0.015 \pm 0.139$ vs. $0.009 \pm 0.104, \mathrm{P}<0.05)$, and cardiovascular causes $(0.014 \pm 0.144$ vs. $0.009 \pm 0.099$, $\mathrm{P}<0.05)$. The five most common diseases for hospitalization 


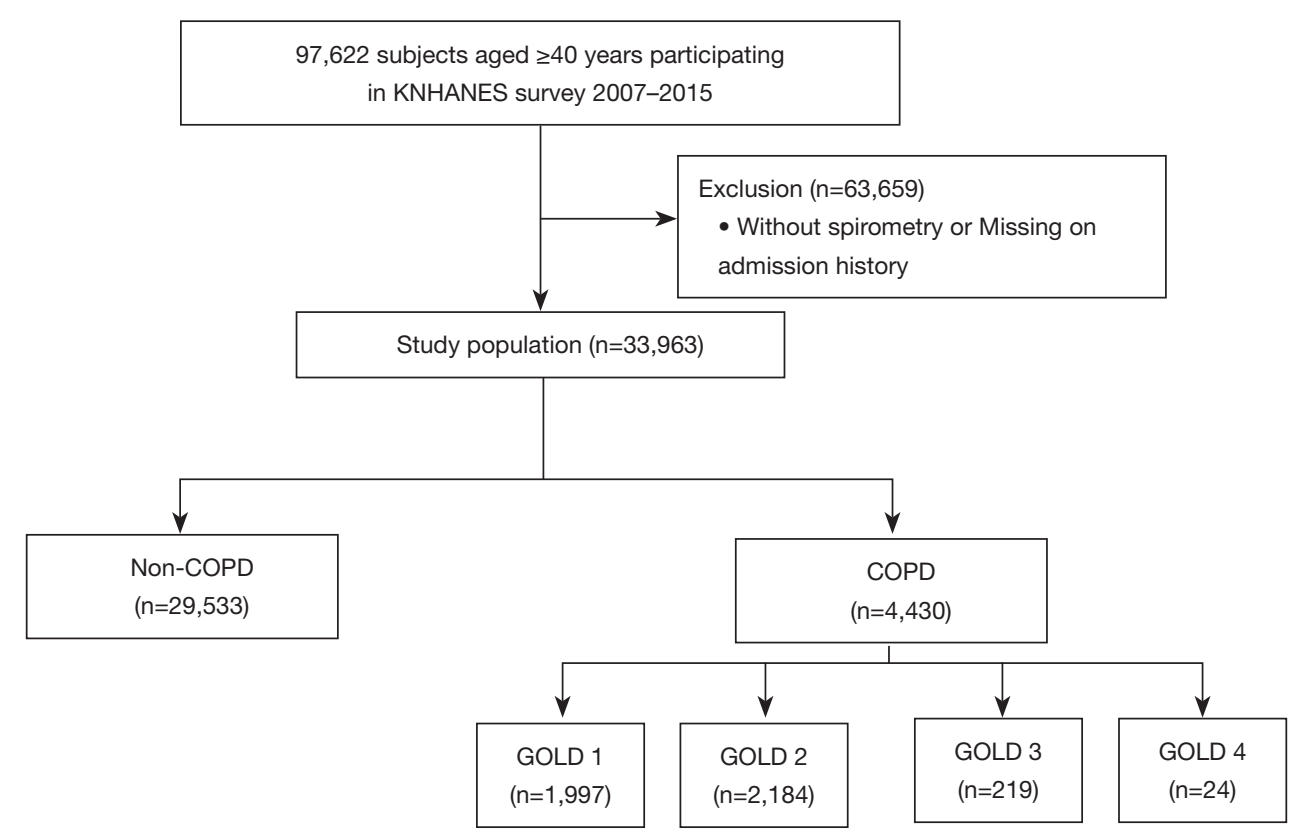

Figure 1 Flow chart representing the selection of the study population. COPD, chronic obstructive pulmonary disease; GOLD, Global Initiative for Chronic Obstructive Lung Disease.

in each category of respiratory disorders, gastrointestinal illnesses, cardiovascular abnormalities, and neoplasm were summarized at a separate table (Table 3).

\section{Hospitalization rate according to GOLD stage}

As the stage of COPD advanced from GOLD 1 to 4 , the admission rate increased for total causes (non-COPD to GOLD $1-4 ; 10.4 \%, 11.5 \%, 13.6 \%, 15.1 \%$, and $25.0 \%$, $\mathrm{P}<0.01$ ) and respiratory causes (Non-COPD to GOLD 1-4; $0.4 \%, 0.5 \%, 1.3 \%, 4.6 \%$, and $12.5 \%, \mathrm{P}<0.01$ ) (Figure 2). Binary logistic regression analysis adjusted for age, sex, smoking status, and household income showed that hospitalization for total causes showed an increasing tendency compared to non-COPD as the stage of COPD advanced (Odds ratio of GOLD 1-4; 1.072, 1.325, 1.365, and 2.630) which was statistically significant in GOLD 2 and GOLD 4. Respiratory admission also showed an increasing tendency in accordance with COPD stage compared to non-COPD (odds ratio for GOLD 1-4; 0.862, 2.649, 8.621, and 26.374), which was statistically significant in GOLD2-4 (Table 4).

\section{Discussion}

The present study evaluated the burden of hospitalization for the number, rate and economic aspect among KNHANES participants for 2007-2015, depending on the presence and severity of COPD. We showed that participants with COPD had a higher rate and frequency of hospitalization in the previous year than those without COPD. For causes of admission, patients with COPD had a higher rates and frequency of hospital admissions than those without COPD for respiratory illnesses, gastrointestinal diseases, and cardiovascular causes. Of note, the admission rate increased for total and respiratory causes as the stage of COPD advanced from GOLD 1 to 4, while the economic status deteriorated in the late stages of COPD.

As expected, patients with COPD had a higher number and rate of hospitalization in the previous year due to respiratory illnesses. In one large population-based study, COPD always occupied the first place in the frequency of hospital admissions for adult patients among four major respiratory diseases such as COPD, pneumonia, lung cancer, and asthma (12). It is well-known that patients with COPD often suffer from multiple comorbidities $(13,14)$. In particular, COPD is associated with increased an risk of cardiovascular morbidities and approximately one-third of COPD deaths are estimated to be due to cardiovascular causes $(15,16)$. Moreover, physical inactivity accompanying COPD frequently leads to functional constipation, 
Table 1 Demographics, pulmonary function, economic status and comorbidities

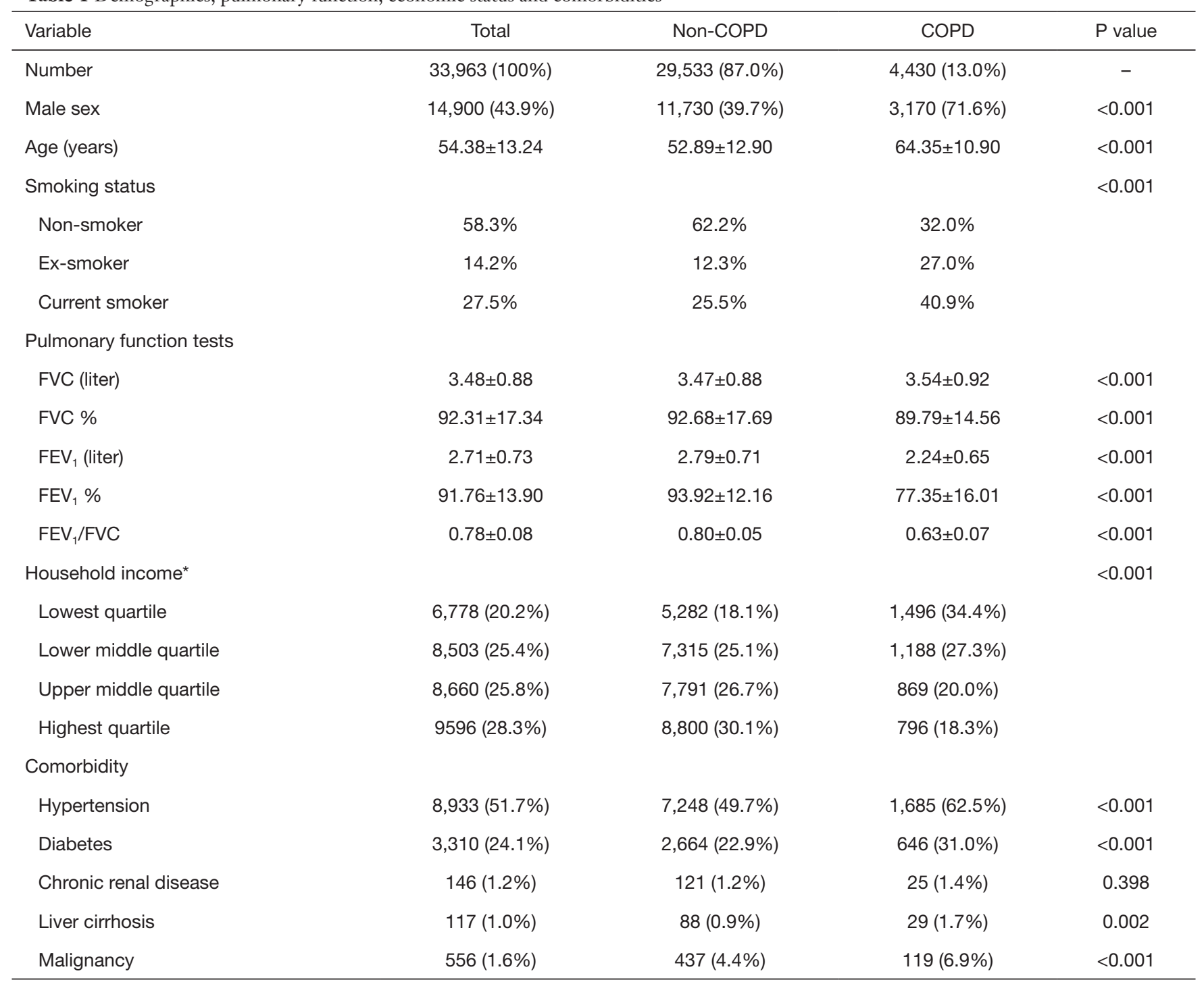

*, household income was measured as combined income from all sources of the respondent and family members and was divided to four quartiles according to criteria of each year.

and the use of inhaled long-acting muscarinic receptor antagonists may cause anti-cholinergic adverse events such as constipation in patients with COPD (17). Corticosteroid use is associated with an increased risk of duodenal ulcer perforation. Because inhaled and systemic corticosteroid are frequently used in the management of COPD, up to $30 \%$ of COPD patients have been found to have peptic ulcers, and COPD frequency in peptic ulcer disease is $2-3$ times the general population (18). This finding is consistent with our results that participants with COPD had more major comorbidities such as hypertension, diabetes, liver cirrhosis, and malignancy than those without COPD (Table 1). Furthermore, our analysis showed that COPD patients were more frequently hospitalized than those without COPD not only due to respiratory causes but also for cardiovascular and gastrointestinal illnesses.

Previous reports have shown that the severity of COPD is associated with the frequency of hospitalization. Patients in the GOLD D category experienced nearly three times the number of exacerbations and COPD-related admissions as those in the GOLD A category (19). The present analysis showed that both acute exacerbation and respiratory 
Table 2 Demographics, pulmonary function, economic status and comorbidities according to the GOLD stage

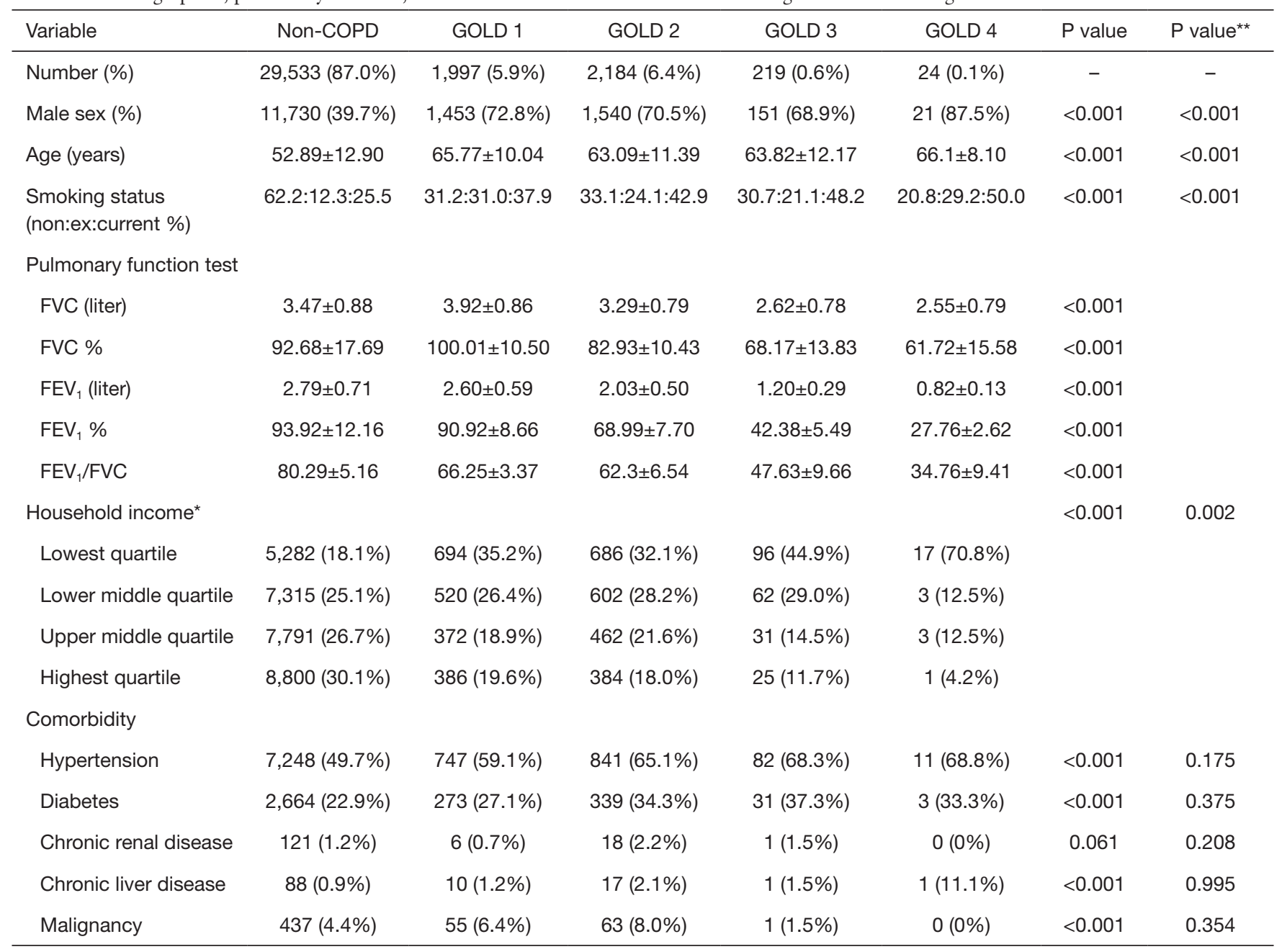

*, household income was measured as combined income from all sources of the respondent and family members and was divided to 4 quartiles according to criteria of each year; ${ }^{* *}$, multivariate linear regression was performed for age, sex, smoking status, household income, and comorbidity. GOLD, Global Initiative for Chronic Obstructive Lung Disease.

illnesses generally increased hospitalization in COPD. Moreover, the total hospitalization rate increased as the stage progressed in COPD (Figure 2).

Patients with COPD suffer from the diseases and their associated financial burden. COPD is a disease of the poor. The Center for Disease Control and Prevention reported that $20.4 \%$ of those with COPD were unable to work compared to $4.8 \%$ without the disease (20). Americans older than 50 years who had COPD were less likely to be employed than those without COPD or those with cancer, heart disease, hypertension, or diabetes (21). Frequent hospitalization places a tremendous financial burden on individuals of low socioeconomic status. Unfortunately, that was what happened in the real world; the hospitalization rate increased as the COPD stage advanced (Table 3, Figure 2), while the lowest quartile of household income increased sharply in the late stages of COPD (Table 2). A striking observation was that the percentile of the lowest quartile of household income in GOLD 4 was $>70 \%$.

The present study has some limitations. First, the KNHANES dataset is a cross-sectional dataset. The positive associations between the number and rates of hospitalization for COPD do not imply causality. Second, although we were able to determine that low economic status was associated with the late stages of COPD, we were unable to determine the underlying causes. Third, the diagnosis of COPD was based on the pre-bronchodilator PFT findings instead of post-bronchodilator ones because it 
Table 3 The five most common diseases of hospitalization in each category of disorder

\begin{tabular}{|c|c|c|c|c|}
\hline & Respiratory illness & Gastrointestinal disease & Cardiovascular disease & Neoplasm \\
\hline 2 & Asthma [25] & Cholelithiasis and cholecystitis [67] & Intracranial hemorrhage [28] & Breast cancer [38] \\
\hline 3 & COPD [20] & Appendix disease [46] & Acute myocardial infarction [25] & Leiomyoma of the uterus [36] \\
\hline 5 & Acute pharyngitis, tonsillitis [5] & Other liver diseases [23] & $\begin{array}{c}\text { Conduction disorder and cardiac } \\
\text { arrhythmia [15] }\end{array}$ & Lung cancer [5] \\
\hline
\end{tabular}

The number in square bracket means the number of hospitalization.

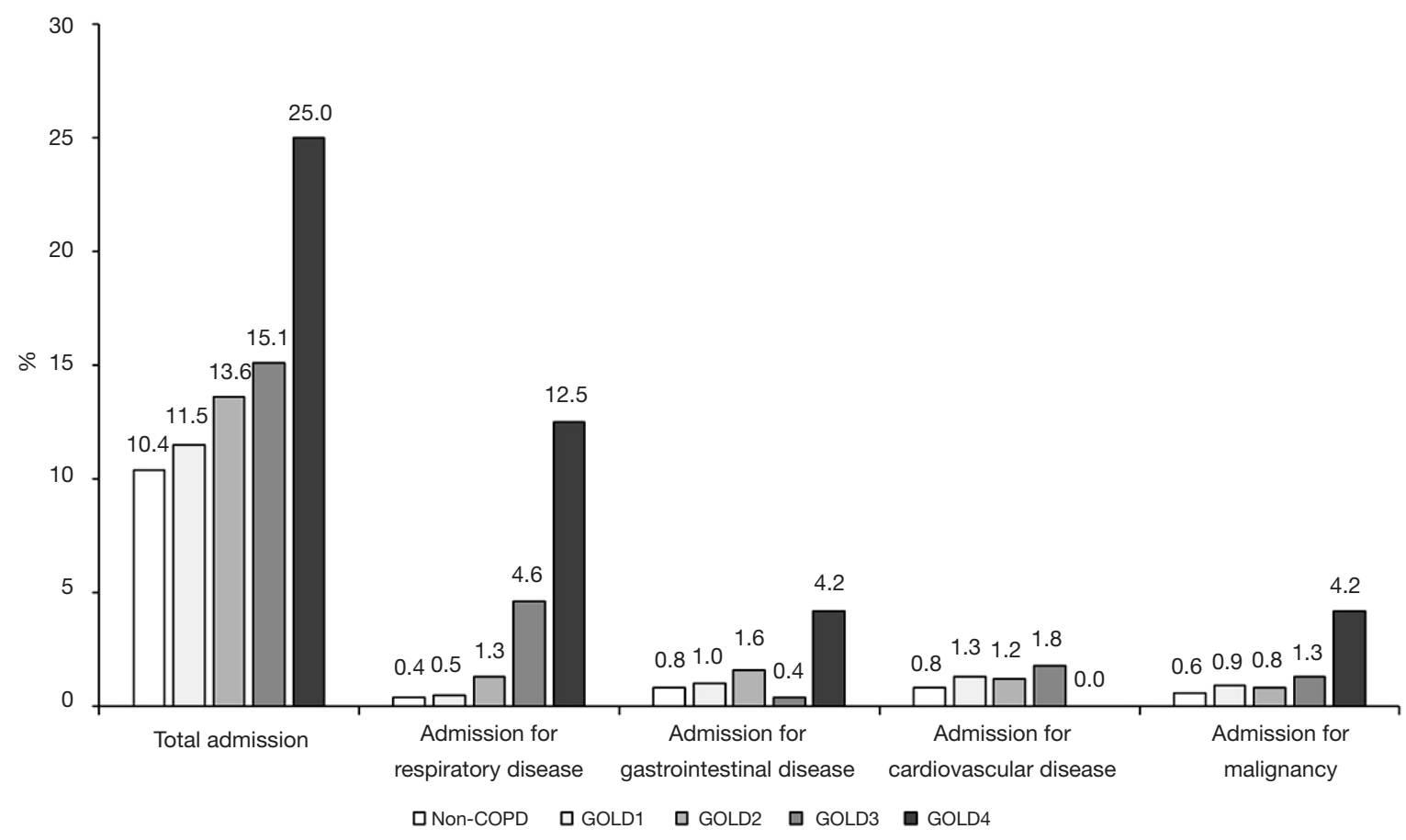

Figure 2 Hospitalization percentage in the previous year for all causes, respiratory diseases, gastrointestinal diseases, cardiovascular diseases, and malignancy according to the GOLD stage. GOLD, Global Initiative for Chronic Obstructive Lung Disease.

was only available in the KNHANES, which is a deviation from GOLD guideline of COPD diagnosis. Nevertheless, to the best of our knowledge, this is the first nation-wide analysis showing that hospitalization increased in the later stages of COPD not only for respiratory and cardiovascular diseases but also for gastrointestinal and all related causes. Moreover, we were able to determine the burden of hospitalization for the number, rate, and economic aspects according to the severity of COPD.

In conclusion, the hospitalization rate increased as the stage of COPD advanced, while the economic status of the patients deteriorated in GOLD stages 3 and 4 . The burden of admission, including the rate, number, and costs increased in the late stages of COPD. 
Table 4 Association between GOLD stages and the risk of hospitalization

\begin{tabular}{|c|c|c|c|c|c|}
\hline Admission & Non-COPD & GOLD1 & GOLD2 & GOLD3 & GOLD4 \\
\hline Odds ratio & 1 & 1.072 & 1.325 & 1.365 & 2.630 \\
\hline $95 \% \mathrm{Cl}$ & & $0.922-1.246$ & $1.157-1.16$ & 0.929-2.007 & $1.040-6.648$ \\
\hline$P$ value & & 0.367 & $<0.001$ & 0.113 & 0.041 \\
\hline Odds ratio & 1 & 0.862 & 2.649 & 8.621 & 26.374 \\
\hline $95 \% \mathrm{Cl}$ & & $0.427-1.740$ & $1.701-4.126$ & $4.218-1.617$ & $7.561-92.004$ \\
\hline$P$ value & & 0.678 & $<0.001$ & $<0.001$ & $<0.001$ \\
\hline Gastrointestinal disease & $246(0.8 \%)$ & $20(1.0 \%)$ & $35(1.6 \%)$ & $1(0.4 \%)$ & $1(4.2 \%)$ \\
\hline$P$ value & & 0.831 & 0.029 & 0.392 & 0.222 \\
\hline Cardiovascular disease & $244(0.8 \%)$ & $26(1.3 \%)$ & 27 (1.2\%) & $4(1.8 \%)$ & $0(0 \%)$ \\
\hline Odds ratio & 1 & 1.117 & 1.115 & 1.587 & - \\
\hline $95 \% \mathrm{Cl}$ & & $0.726-1.719$ & $0.733-1.697$ & $0.579-4.53$ & - \\
\hline$P$ value & & 0.615 & 0.611 & 0.369 & 0.998 \\
\hline Neoplasm & $193(0.6 \%)$ & $19(0.9 \%)$ & $19(0.8 \%)$ & $3(1.3 \%)$ & $1(4.2 \%)$ \\
\hline Odds ratio & 1 & 1.333 & 1.266 & 2.278 & 7.579 \\
\hline $95 \% \mathrm{Cl}$ & & $0.788-2.254$ & $0.754-2.127$ & $0.714-7.269$ & $1.004-57.215$ \\
\hline
\end{tabular}

In this binary logistic regression analysis, results were adjusted by age, sex, smoking status, and household income. GOLD, Global Initiative for Chronic Obstructive Lung Disease.

\section{Acknowledgments}

Funding: This study was supported by Pfizer Grant W1221325. Except the financial support, Pfizer did not involve any process of the present study including the design of the study to the writing of the manuscript.

\section{Footnote}

Reporting Checklist: The authors have completed the STROBE reporting checklist. Available at http://dx.doi. org/10.21037/jtd-20-2683

Peer Review File: Available at http://dx.doi.org/10.21037/jtd20-2683

Conflicts of Interest: All authors have completed the ICMJE uniform disclosure form (available at http://dx.doi. org/10.21037/jtd-20-2683). The authors have no conflicts of interest to declare.

Ethical Statement: The authors are accountable for all aspects of the work in ensuring that questions related to the accuracy or integrity of any part of the work are appropriately investigated and resolved. The study was conducted in accordance with the Declaration of Helsinki (as revised in 2013). All KNHANES survey protocols were approved by the KCDC institutional review board (201501-02-6C). Written informed consent was obtained from all the participants.

Open Access Statement: This is an Open Access article distributed in accordance with the Creative Commons 
Attribution-NonCommercial-NoDerivs 4.0 International License (CC BY-NC-ND 4.0), which permits the noncommercial replication and distribution of the article with the strict proviso that no changes or edits are made and the original work is properly cited (including links to both the formal publication through the relevant DOI and the license). See: https://creativecommons.org/licenses/by-nc-nd/4.0/.

\section{References}

1. GBD 2015 Chronic Respiratory Disease Collaborators. Global, regional, and national deaths, prevalence, disability-adjusted life years, and years lived with disability for chronic obstructive pulmonary disease and asthma, 1990-2015: a systematic analysis for the Global Burden of Disease Study 2015. Lancet Respir Med 2017;5:691-706.

2. Hwang YI, Park YB, Yoo KH. Recent trends in the prevalence of chronic obstructive pulmonary disease in Korea. Tuberc Respir Dis (Seoul) 2017;80:226-9.

3. Kochanek KD, Murphy SL, Xu J, et al. Mortality in the United States, 2016. Atlanta, Georgia: Centers for Disease Control and Prevention, 2017.

4. Park SC, Kim DW, Park EC, et al. Mortality of patients with chronic obstructive pulmonary disease: a nationwide population based cohort study. Korean J Intern Med 2019;34:1272-8.

5. Criner RN, Han MK. COPD Care in the 21st Century: A Public Health Priority. Respir Care 2018;63:591-600.

6. Kim C, Kim Y, Wang D, et al. Direct and Indirect Costs of Chronic Obstructive Pulmonary Disease in Korea. Tuberc Respir Dis (Seoul) 2019;82:27-34.

7. Kim C, Yoo KH, Rhee CK, et al. Health care use and economic burden of patients with diagnosed chronic obstructive pulmonary disease in Korea. Int J Tuberc Lung Dis 2014;18:737-43.

8. Pleasants RA, Riley IL, Mannino DM. Defining and targeting health disparities in chronic obstructive pulmonary disease. Int J Chron Obstruct Pulmon Dis 2016;11:2475-96.

9. Kumbhare SD, Beiko T, Wilcox SR, et al. Characteristics of COPD patients using United States emergency care or hospitalization. COPD 2016;3:539-48.

10. Vespasiani-Gentilucci U, Pedone C, Muley-Vilamu $\mathrm{M}$, et al. The pharmacological treatment of chronic comorbidities in COPD: mind the gap! Pulm Pharmacol
Ther 2018;51:48-58.

11. Kweon S, Kim Y, Jang MJ, et al. Data resource profile: the Korea National Health and Nutrition Examination Survey (KNHANES). Int J Epidemiol 2014;43:69-77.

12. Liu H, Wang N, Chen W, et al. Hospitalization Trends in Adult Patients With COPD and Other Respiratory Diseases in Northeast China From 2005 to 2015. Biomed Res Int 2018;2018:1060497.

13. Triest FJJ, Franssen FME, Reynaert N, et al. DiseaseSpecific Comorbidity Clusters in COPD and Accelerated Aging. J Clin Med 2019;8:511.

14. Gershon AS, Mecredy GC, Guan J, et al. Quantifying Comorbidity in Individuals With COPD: A Population Study. Eur Respir J 2015;45:51-9.

15. Sin DD, Anthonisen NR, Soriano JB, et al. Mortality in COPD: role of comorbidities. Eur Respir J 2006;28:1245-57.

16. Donaldson GC, Hurst JR, Smith CJ, et al. Increased risk of myocardial infarction and stroke following exacerbation of COPD. Chest 2010;137:1091-7.

17. Hanlon P, Nicholl BI, Jani BD, et al. Examining patterns of multimorbidity, polypharmacy and risk of adverse drug reactions in chronic obstructive pulmonary disease: a crosssectional UK Biobank study. BMJ Open 2018;8:e018404.

18. Nguyen MHK, Isaac KM, Dougherty R. Gastrointestinal perforation: relation to corticosteroid use and COPD a case report. J Community Hosp Intern Med Perspect 2016;6:32129.

19. Merinopoulou E, Raluy-Callado M, Ramagopalan S, et al. COPD exacerbations by disease severity in England. Int J Chron Obstruct Pulmon Dis 2016;11:697-709.

20. Wheaton AG, Cunningham TJ, Ford ES, et al. Employment and activity limitations among adults with chronic obstructive pulmonary disease-United States, 2013. MMWR Morb Mortal Wkly Rep 2015;64:289-95.

21. Thornton Snider J, Romley JA, Wong KS, et al. The disability burden of COPD. COPD 2012;9:513-21.

Cite this article as: Kang HK, Jung JW, Kang MJ, Kim DK, Choi H, Cho YJ, Jang SH, Lee CH, Oh YM, Park J, Kim JY. Hospitalization increases while economic status deteriorates in late stages of chronic obstructive pulmonary disease: the Korean National Health and Nutrition Examination Survey for 2007-2015. J Thorac Dis 2021;13(4):2160-2168. doi: 10.21037/jtd20-2683 